Documentation et bibliothèques

DOCUMENTATION BIBLIOTHEQUES

\title{
La Bibliothèque de France, bibliothèque de toutes les
} recherches

\section{The Bibliothèque de France, a Library for all Types of Research La Biblioteca de Francia, biblioteca de todas las investigaciones}

\section{Dominique Jamet}

Volume 39, numéro 2, avril-juin 1993

Les bibliothèques nationales

URI : https://id.erudit.org/iderudit/1028736ar

DOI : https://doi.org/10.7202/1028736ar

Aller au sommaire du numéro

Éditeur(s)

Association pour l'avancement des sciences et des techniques de la documentation (ASTED)

ISSN

0315-2340 (imprimé)

2291-8949 (numérique)

Découvrir la revue

Citer cet article

Jamet, D. (1993). La Bibliothèque de France, bibliothèque de toutes les recherches. Documentation et bibliothèques, 39(2), 59-64.

https://doi.org/10.7202/1028736ar
Résumé de l'article

La Bibliothèque de France, dont la construction est en cours, héritera des collections et des missions de la Bibliothèque nationale actuelle. Dotée d'une architecture à la mesure du projet, la nouvelle Bibliothèque nationale offrira des aménagements propices à tous les types de recherches et développera ses échanges avec les bibliothèques étrangères. L'ouverture se fera progressivement à compter de janvier 1996.
Tous droits réservés (C) Association pour l'avancement des sciences et des techniques de la documentation (ASTED), 1993
Ce document est protégé par la loi sur le droit d'auteur. L'utilisation des services d'Érudit (y compris la reproduction) est assujettie à sa politique d'utilisation que vous pouvez consulter en ligne. 


\title{
La Bibliothèque de France, bibliothèque de toutes les recherches
}

\author{
Dominique Jamet \\ Président de l'Établissement public de la Bibliothèque de France
}

La Bibliothèque de France, dont la construction est en cours, héritera des collections et des missions de la Bibliothèque nationale actuelle. Dotée d'une architecture à la mesure du projet, la nouvelle Bibliothèque nationale offrira des aménagements propices à tous les types de recherches et développera ses échanges avec les bibliothèques étrangères. L'ouverture se fera progressivement à compter de janvier 1996.

\section{The Bibliothèque de France, a Library for all Types of Research}

The Bibliothèque de France, currently under construction, inherited the collections and the mission of the present Bibliotheque nationale. The architecture reflects the grandeur of the project and the new national library will offer a layout conducive to all types of research. Exchanges with foreign libraries will be created. The library will begin operations in January 1996 and progressively extend its services.

\section{La Biblioteca de Francia, biblioteca de todas las investigaciones}

La Biblioteca de Francia, la cualestá actualmente en construcción, heredará las colecciones y las funciones de la Biblioteca Nacional actual. La nueva Biblioteca Nacional, dotada de una arquitectura apropiada al proyecto, ofrecerá instalaciones propicias para todos los tipos de investigaciones y desarrollará sus intercambios con las bibliotecas extranjeras. La inauguración se hará progresivamente a partir de enero de 1996.

\section{Enjeux de la Bibliothèque de France}

\section{Un projet national}

La décision de doter la France d'une nouvelle Bibliothèque Nationale, bibliotheqque «qui puisse prendre en compte toutes les données du savoir dans toutes les disciplines, et surtout qui puisse communiquer ce savoir à l'ensemble de ceux qui cherchent, qui étudient, qui ont besoin d'apprendrew a été prise, ou du moins rendue publique, le 14 juillet 1988, par François Mitterrand. Dès lors qu'elle émanait du chef de l'État, que l'État, par le truchement d'un établissement public constructeur et sur la base d'un financement intégralement public, en était le maître d'ouvrage unique, compte tenu, enfin, de l'ambition affichée, l'entreprise pouvait passer pour éminemment «politique». Pour autant, il serait aussi mal venu d'y voir, comme cela put être dit à l'occasion de telle ou telle polémique, le fait d'un caprice «personnel» - et naturellement "pharaonique» - qu'on ne sait quelle intention partisane, électoraliste encore moins. Un projet comme celui-là, porteur d'une visée à long terme, n'est ni de droite ni de gauche. II doit transcender les cliva- ges et les alternances. La réalisation, l'ouverture et la réussite de la Bibliothèque de France constituent un objectif d'intérêt national.

La création, en cette fin de $X X^{e}$ siècle, d'une bibliothèque de recherche plus vaste plus complète, plus moderne, plus ouverte, plus généreuse que l'actuelle Nationale', relève d'évidentes nécessités techniques, sociales et intellectuelles, et répond à une volonté, on pourrait même dire à une philosophie, de démocratisation de la culture.

\section{Des carences anciennes}

À l'origine du projet actuel, que trouve-t-on en effet? Le constat de carence, une carence qui tient moins à l'impéritie des hommes qu'à l'insuffisance des moyens et aux restrictions, aux renoncements, aux abandons subséquents d'une bibliothèque nationale certes prestigieuse et riche en fonds anciens, mais n'offrant qu'à un petit nombre d'usagers ( 400 places en salle de lecture des imprimés, 200 places en salle de lecture des périodiques) appartenant à des catégories sociales très délimitées ( $85 \%$ d'universitaires, $76 \%$ d'historiens et d'historiens de l'art, $76 \%$ de Parisiens contre $12 \%$ de provinciaux et $12 \%$ d'étrangers) des collections insuffisamment différenciées (le dépót légal et le faible volume des acquisitions ne permettent ni la constitution ni l'actualisation d'un fonds réellement encyclopédique) conservées, de plus, à l'intérieur de locaux inadaptés et saturés, dans des conditions insatisfaisantes (trois millions d'ouvrages sont d'ores et déjà incommunicables ou en voie de dégradation rapide). La Bibliothèque nationale, hier à la mesure d'une France de 38 millions d'habitants, dont 5000 chaque année accédaient à l'enseignement supérieur, n'est plus proportionnée aux dimensions d'un pays de 60 millions d'habitants où $80 \%$ de chaque classe d'âge atteignent le niveau du baccalauréat, et où l'on compte 15000 titulaires du doctorat.

\section{Des besoins nouveaux}

Dès lors, de quoi s'agit-il? De la même manière que Malraux avait émis le voeu, en matière artistique, de urendre accessibles les oeuvres capitales de l'humanité, et d'abord de la France, au plus grand nombre de Français", l'ambition

1. La Bibliothèque nationale française. 
de la Bibliothèque de France est d'assurer dans les meilleures conditions possibles l'accès du plus grand nombre, sur place et à distance, aux sources du savoir et à la lecture savante, bref d'offrir à tous, en tous points, «les clés du trésor». II s'agit de fournir à la société contemporaine un instrument scientifique adapté aux demandes formulées et potentielles. $\mathrm{Au}$ travers de bien des vicissitudes, l'entreprise en cours aura constamment visé à mettre en harmonie la logique des besoins nouveaux et les moyens de la technique actuelle.

Socialement, on passe du restreint à l'ouvert, du caché au transparent, de l'élitiste, pour quelques-uns, à l'élitaire pour tous. Une bibliothèque nationale moderne ne saurait se résumer à un conservatoire du patrimoine nimême fonctionner comme un sanctuaire de première classe réservé par ses grands-prêtres à l'usage de quelques mandarins à six boutons. C'est un outil scientifique majeur qu'il convient de mettre à la disposition de la collectivité nationale - d'abord de la communauté des chercheurs, mais aussi de toutes les catégories socioprofessionnelles intéressées, juristes, économistes, scientifiques (au sens étroit du mot), éditeurs, journalistes, etc., - un atout majeur dans la compétition internationale qui, bien souvent, est à la base, une compétition intellectuelle. Quantitativement, on passe, sur le site, de 600 à 3400 places de lecture et des 300000 entrées annuelles de Richelieu à 3000000 d'entrées. C'est un premier changement d'échelle, auquel la possibilité de consulter à distance le catalogue, puis une fraction croissante des collections, ajoutera un deuxième saut quantitatif.

Entre 1988 et 1993, comme il est normal, et comme il était prévu dès l'origine, le projet aura connu nombre de précisions, de modifications, d'adaptations, d'améliorations. En tout état de cause, un pas décisif avait été accompli lorsque, en août 1989, conformément à l'instante demande d'une grande majorité de chercheurs, le principe fut arrêté de transférer à Tolbiac la totalité des fonds d'imprimés, de périodiques et de documents audiovisuels conservés rue de Richelieu. Dece fait, le projet ne changeait pas seulement de dimension, mais de signification. II impliquait désormais très clairement la fusion entre une bibliothèque patrimoniale inscrite depuis longtemps dans notre tradition intellectuelle et une bibliothèque électronique que certains présentaient encore comme utopique alors que cette utopie est en train de devenir réalité ça et là. C'était encore une bibliothèque d'études et de recherches d'un modèle connu, et déjà une bibliothèque immatérielle fonctionnant en réseau, donc «d'un type nouveau», la Bibliothèque nationale, mais transfigurée.

\section{Des ambitions nouvelles}

II s'ensuit que la Bibliothèque de France recueillera et assumera, avec les mêmes soins et les mêmes scrupules qu'aujourd'hui, mais, souhaitons-le, avec des moyens accrus, l'héritage des collections et des missions de la Bibliothèque nationale. II lui reviendra donc d'amasser, d'accroître, de conserver et de préserver le patrimoine de l'écrit. Mais elle développera également des ambitions nouvelles correspondant à de nouvelles exigences. Elle s'ouvrira à des supports nouveaux, à des techniques nouvelles, à de nouvelles pratiques. Une bibliothèque nationale moderne ne saurait plus longtemps ignorer la prégnance de l'audiovisuel dans notre société, sa présence dans notre histoire, son utilité pour la recherche. Une bibliothèque nationale moderne, sous peine de se cantonner dans un gallocentrisme dérisoire, ne peut désormais se passer de la production étrangère, ni faire l'impasse sur tel ou tel domaine scientifique, dans un temps où les cultures dialoguent, où les disciplines s'interpénètrent. Une bibliothèque nationale moderne ne peut ignorer les fantastiques facilités qu'offre l'électronique au rassemblement, au stockage, à la circulation de l'information. Alors que la numérisation et la lecture assistée par ordinateur ouvrent une ère nouvelle dans la transmission, la diffusion et l'utilisation du savoir, on n'imagine pas plus une bibliothèque sans l'informatique qu'une maison sans eau courante et sans l'électricité. Une bibliothèque nationale moderne peut, donc elle doit, offrir l'accès le plus libre et le plus large possible, non à la lecture - ce n'est pas de son ressort -mais à linformation, à la documentation, aux grands gisements de connaissance.
Ainsi incombera-t-il à la Bibliothèque de France de couvrir tous les champs disciplinaires, d'être à la disposition de tous, sur place et à distance, de se trouver enfin, élément fort d'un réseau de communication multimédias, au coeur d'un dispositif qui doit inclure tous les moyens de lecture, sur le territoire français, puis au-delà des frontières.

\section{Une architecture a la dimension du projet}

\section{Un concours}

Le choix de l'architecte de la Bibliothèque de France s'est opéré à l'issue d'un concours international d'idées sur invitation, organisé par l'Association pour la Bibliothèque de France, en étroite collaboration avec l'Union internationale des architectes. En juillet 1989, le jury international que présidait I.M. Pei a retenu quatre projets, en distinguant particulièrement celui de Dominique Perrault qui est choisi par le Président de la République le 21 août 1989 pour construire la Bibliothèque de France à Tolbiac.

\section{Une architecture}

Le bâtiment dessiné par Dominique Perrault se compose schématiquement d'un socle évidé dans sa partie centrale et de quatre tours d'angle en forme de livre ouvert. Les quatre tours, hautes de $79 \mathrm{~m}$, abriteront chacune, derrière leur peau de verre, sept étages de bureaux protégés par des volets de bois mobiles et onze étages de magasins protégés par une paroi fixe de même aspect extérieur, mais composée de matériaux isolants. La tour nord-est comportera un belvédère accessible au public.

Le socle, relié au parc de Bercy, sur l'autre rive, par une passerelle piétonne, formera une esplanade unique aux dimensions de la place de la Concorde, accessible du quai par de grands emmarchements agrémentés, à espaces réguliers, de fontaines. Cette esplanade sera recouverte d'un sol de bois qu'on parcourra pour gagner les entrées de la Bibliothèque. Celleci ne se signalera d'abord que par la cime des arbres affleurant d'un jardin comparable, par sa taille, à celui du Palais-Royal, aménagé dans la partie centrale. Ce jardin central est ceinturé sur deuxniveaux par les salles de lecture. 


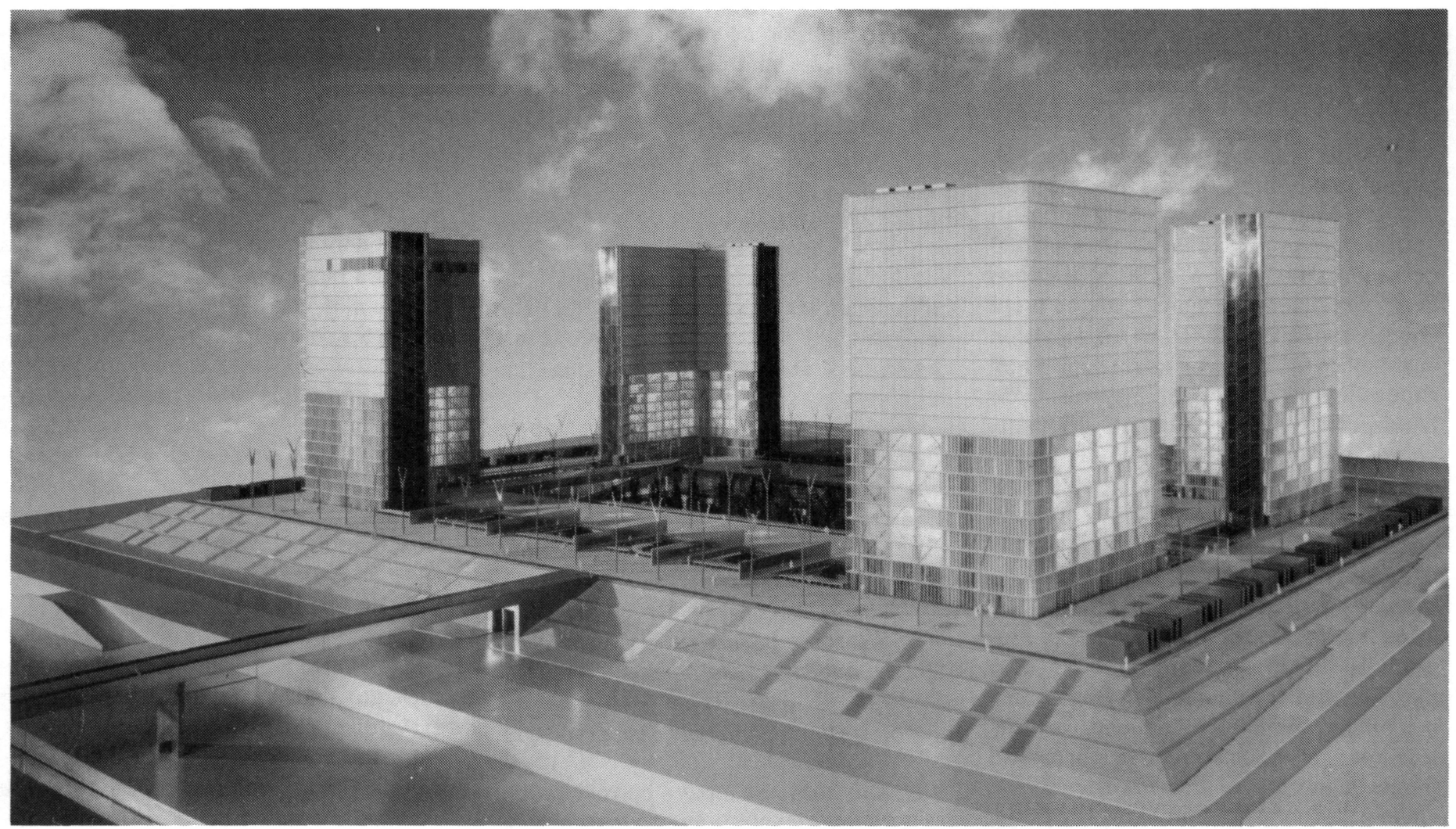

Maquette de la Bibliothèque de France (C) Maurer P.

\section{Une grande réalisation}

Aujourd'hui, l'état d'avancement des travaux est de $60 \%$ des surfaces, soit plus de $200000 \mathrm{~m}^{2}$. Le socle est déjà accompli en grande partie. Les magasins du socle sont réalisésà $100 \%$. Lebâtiment sera achevé en novembre 1993 en ce qui concernele grosoeuvre et en juin 1994, pour les façades. Les lots techniques seront terminés à la fin de 1994. Pour les équipements etles mobiliers, la phase de réalisation commenceen mars 1994 et leur installation sera terminée en octobre 1995.

\section{Une bibliothèque de recherche pour tous}

\section{La bibliothèque de toutes les re- cherches}

La Bibliothèque de France, à Tolbiac, constituera bien, sur deux étages, une grande bibliothèque de recherche. L'unité de la Bibliothèque est exprimée par l'homologie dans l'organisation des deux niveaux: au rez-de-jardin, la bibliothèque de recherche spécialisée, en haut-de-jardin, la bibliothèque publique de recherche.

\section{La bibliothèque de recherche spécialisée}

D'une part, la Bibliothèque de France demeurera en effet, comme la Nationale actuelle, avec un meilleur confort, avec une plus grande ampleur, la bibliothèque des spécialistes, des professionnels de recherche, essentiellement des universitaires avec une part importante de chercheurs étrangers. Ce public sera toutefois élargi à des chercheurs de nouvelles disciplines (économistes, juristes, scientifiques) dans la mesure où la nouvelle Bibliothèque nationale constituera de véritables collections dans ces disciplines et ne se limitera pas aux humanités. Ce public de la bibliothèque de recherche spécialisée est estimé à près de 3000 personnes par jour.

Offrant 2030 places de lecture et une collection en libre accès qui atteindra à terme 480000 volumes, la bibliothèque de recherche spécialisée accueillera un public strictement accrédité. Ce public de chercheurs recevra tout le renfort des moyens nouveaux mis à sa disposition: lecteurs de microformes, bornes de consultation des catalogues, postes de lec- ture assistée par ordinateur reliés à une imprimante, postes de consultation audiovisuelle...

La bibliothèque de recherche spécialisée sera composée des espaces suivants:

\section{- Un service de recherche bibliographique}

- Quatre départements thématiques: sciences et techniques; littératures et art; sciences politiques, juridiques et économiques; philosophie, histoire, sciences de l'homme et de la société. Ces quatre départements thématiques fonctionneront sur un schéma commun. Dans chaque département, les banques de salle permettront la communication des documents, dispenseront éventuellement au lecteur une assistance et serviront d'antenne du service de reproduction. Surplombant les salles, des loges, petits bureaux individuels et fermés, regroupés sur des mezzanines, permettrontà ceuxquienont besoin des'isoler et de conserver leurs documents de travail pour une durée d'une demi-journée à deux semaines. Quarante postes de consultation audiovisuelle seront répartis dans 
ces quatre départements pour permettre la consultation des images et des sons en rapport avec les disciplines concernées.

- Le département de límage et du son permettra aux chercheurs d'avoir accès à 720000 heures de documents sonores, 150000 heures d'images animées, 44000 documents multimédia, 1200000 images fixes numérisées auxquelles s'ajouteront chaque année 12000 heures de télévision et autant de radio provenant du dépót légal recueilli par l'Institut national de l'Audiovisuel. Les documents originaux pourront être consultés sur demande dans des cabines spécialisées. De petites salles de projection permettront aux groupes de visionner des documents dans les conditions réelles de leur utilisation.

- La réserve des documents rares et précieux. Elle sera l'héritière de la Réserve de la Bibliothèque nationale qui rassemble incunables, éditions originales, reliures exceptionnelles, exemplaires sur grand papier ou aux provenances prestigieuses. Cette collection sera portée de 150000 à 300000 documents en 1996 auxquels s'ajouteront 12000 usuels en salle.

- La salle de documentation sur le livre et la lecture pour tous ceux qu'intéressent spécifiquement l'histoire et l'actualité du livre, de ses techniques de fabrication, ainsi que des pratiques de lecture.

\section{La bibliothèque publique de recherche}

D'autre part, bibliothèque publique de recherche, la Bibliothèque de France n'a jamais été conçue comme une BPI bis $^{2}$ ou comme une bibliothèque universitaire de plus. Elle n'a pas vocation à l'être. $\mathrm{Ni}$ superflue par rapport à la $\mathrm{BPI}$, ni redondante par rapport à d'autres types de bibliothèques, son rôle ne sera pas d'accueillir des étudiants en peine de places et de manuels pour les accompagner dans leur cursus et pas davantage de satisfaire des besoins de culture de base, de lecture de loisirs, de formation.

Elle offrira en revanche à tous les membres des professions intellectuelles comme à ceux qui, sans leur appartenir, doivent effectuer à un moment quelconque de leur vie professionnelle ou personnelle une recherche relevant d'un processus d'élaboration, d'actualisation ou de transmission de savoir, des fonds ou des services introuvables ailleurs. On trouvera à la Bibliothèque de France, dans les salles de lecture de la bibliothèque publique de recherche un appareil bibliographique, une collection en libre accès encyclopédique et de haut niveau, un apport de documents audiovisuels et un fonds de documents reproduits fournissant un tel accès. Le public de cette bibliothèque publique de recherche est estimé à 4500 personnes par jour.

Ouverte à tous les publics, après délivrance d'une carte par le personnel d'accueil, la bibliothèque publique de recherche offrira 1556 places de lecture et de consultation. Elle sera composée des services suivants:

- Une salle de lecture de la presse et de documentation sur la presse.

\section{- Une salle d'orientation bibliographique.}

- Quatre salles de lecture correspondant aux départements thématiques de la bibliothèque de recherche spécialisée: sciences et techniques; littératures et art; sciences politiques, juridiques et économiques; philosophie, histoire, sciences de l'homme et de la société. Chaque salle sera structurée de façon identique et comprendra un espace présentant des fonds permanents, un espace voué aux nouveautés, un espace voué au patrimoine, présentant les richesses méconnues du fonds patrimonial, notamment sous forme de reproductions. L'espace patrimonial accueillera en particulier de véritables bibliothèques reconstituées autour d'un thème, d'une discipline, d'une recherche, d'un auteur. Par exemple la Bibliothèque de Stendhal, telle que nous permet de la concevoir fidèlement sa correspondance, ou encore la bibliothèque d'une école laïque en pays minier, à la veille de la seconde guerre mondiale.

\section{La culture pour tous}

En dehors de la consultation en salle, la réalité du livre, comme des autres supports, s'éprouve par le recours à des expositions, des lectures et des conférences, des débats et colloques, des produits d'édition, des projections audiovisuelles et des actions pédagogiques.
La Bibliothèque de France accueillera également le public venu visiter les expositions proposées, assister aux colloques et aux conférences qu'elle organisera, s'initier, gråce à l'espace pédagogique, pour les collégiens, et lycéens accompagnés d'un de leurs professeurs, au fonctionnement de la Bibliothèque. Ces activités culturelles s'adresseront à tous les publics, des jeunes désireux d'apprendre à connaître la vie d'une bibliothèque patrimoniale aux chercheurs les plus érudits, habitués des rencontres scientifiques de haut niveau. Le nombre de visiteurs de ce type est de 4500 par jour. Toutes ces activités seront concentrées en haut-de-jardin, au même niveau que la bibliothèque publique de recherche.

\section{Le coeur de réseau}

Le terrain de sept hectares et demi sur lequel s'édifie la Bibliothèque de France est situé sur la rive gauche de la Seine, entre les ponts de Bercy et de Tolbiac, dans le XIII ${ }^{\circ}$ arrondissement de Paris. II se trouve que la construction de la Bibliothèque de France constitue un élément fort de la restructuration $\mathrm{du} \mathrm{XIII}{ }^{\circ}$ arrondissement et s'inscrit dans le cadre de la régénération de l'Est parisien. Pour autant, cette localisation parisienne du siège social d'une institution de service public ne comporte pas que celle-ci soit plus "parisienne» dans son esprit que par exemple la Poste ou la $\mathrm{SNCF}^{3}$. L'objectif de la Bibliothèque de France est d'être vraiment nationale. Cet objectif se concrétise déjà par la mise en route du catalogue collectif informatisé qui regroupera sous la forme d'une banque de données unique consultable à distance les six millions de notices émanant de la Bibliothèque nationale et sept millions de notices représentant la richesse documentaire de 51 bibliothèques municipales, de 31 bibliothèques universitaires et de divers instituts spécialisés. Un appel de candidatures a d'autre part été

2. Ouverte en 1979 , la Bibliothèque publique d'information située à l'intérieur du Centre Georges Pompidou est une bibliothèque de lecture publique ouverte à tous sans formalités. Victime de son succès, cette bibliothèque multimédia est la plupart du temps saturée.

3. Société nationale des chemins de fer français. 


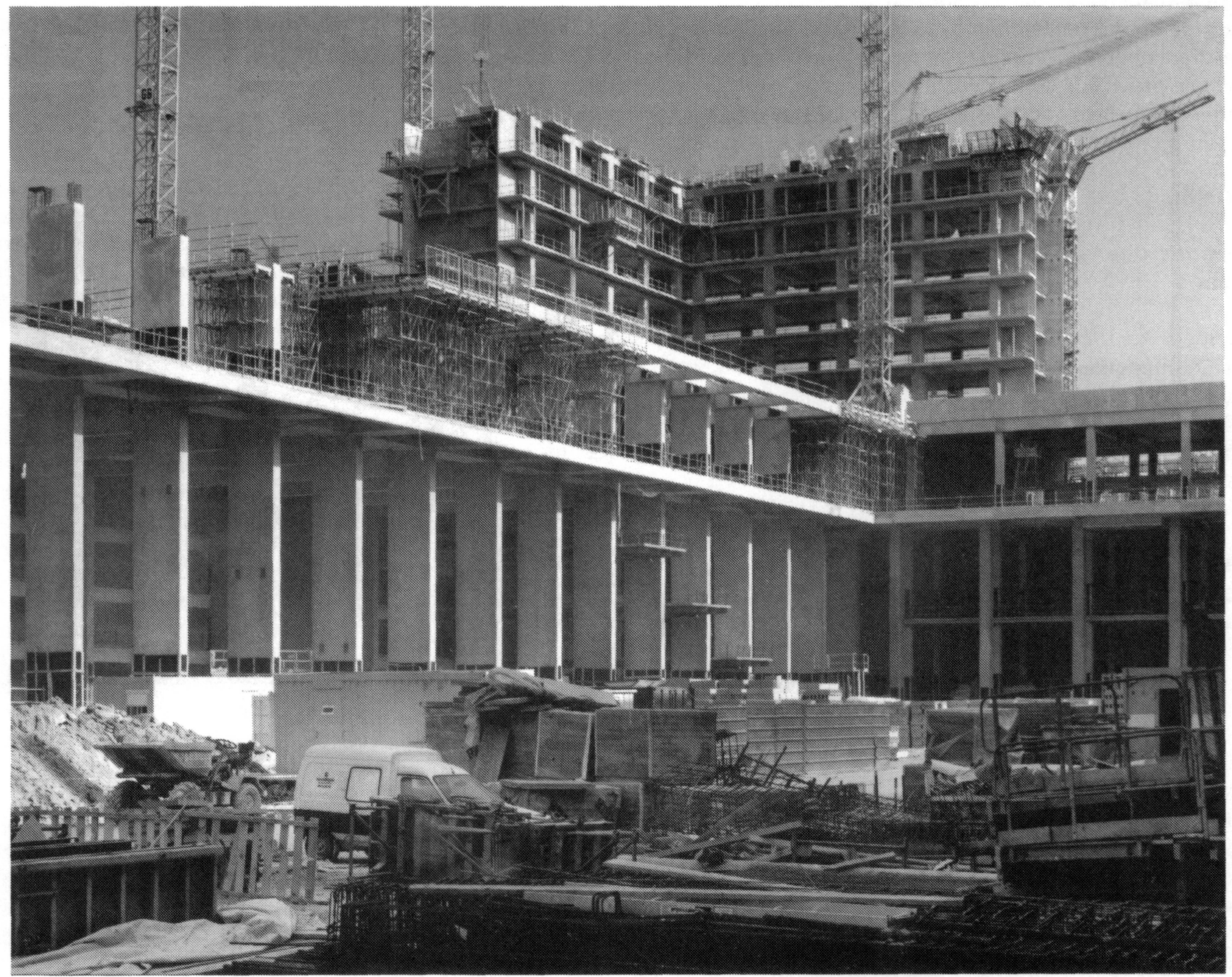

Vue partielle de l'état des travaux au 31 mars 1993. Coustard A.

lancé en direction des centres documentaires régionaux qui, sous la dénomination de «pôles associés», sur la base d'une répartition des crédits d'acquisition et d'une spécialisation, deviendront les partenaires à part entière de la Bibliothèque de France. Enfin la numérisation mettra des ressources documentaires progressivement croissantes à la disposition de tous les usagers du réseau.

\section{La coopération internationale}

Les relations avec les bibliothèques étrangères

Reprenant en les amplifiant les relations qu'entretient la Nationale avec les institutions étrangères, la Bibliothèque de France développera une politique de coopération avec les grandes bibliothèques étrangères nationales (Library of Congress, British Library...) ou universitaires. Des moyens nouveaux dégagés sur les crédits d'acquisitions permettront d'intensifier et d'améliorer la qualité des échanges internationaux de documents.

La Bibliothèque de France s'oriente vers deux voies. Elle souhaite d'une part développer les échanges de services, comme l'échange d'informations bibliographiques et la fourniture de documents à distance. D'autre part, elle considère comme une nécessité le partage des travaux de recherche en bibliothéconomie, dans le cadre de pro- grammes européens ou de programmes bilatéraux.

La Bibliothèque de France apportera de plus sa contribution aux grands programmes internationaux (accès universel aux publications, conservation) conduits par I'UNESCO et I'IFLA. Elle participera activement aux travaux internationaux de normalisation.

\section{La question spécifique de la fran- cophonie}

En ce qui concerne le rayonnement de la francophonie, la Bibliothèque de France mènera des actions de coopération francophone, déjà amorcées par la Bibliothèque nationale, avec les grandes 
bibliothèques étrangères d'expression française, en Amérique du Nord, sur le continent africain ou en Asie du Sud-Est. Des accords seront recherchés avec les éditeurs francophones. Enfin, l'espace francophone prévu sur le site constituera une vitrine des activités éditoriales et culturelles du monde francophone.

\section{Conclusion}

\section{Encore des arbitrages néces- saires}

Compte tenu, notamment, de l'importance numérique, de la diversité des publics attendus et des créneaux de disponibilité qui, pour beaucoup de groupes sociaux, sont peu compatibles avec les horaires d'ouverture habituels aux bibliothèques, il apparaît souhaitable que l'amplitude des horaires de fonctionnement de la Bibliothèque de France excède les normes courantes. L'Établissement public préconise pour sa part comme un minimum incompressible une ouverture de 12 heures par jour, 6 jours par semaine, 300 jours par an.

II importe en effet que l'accès à la Bibliothèque de France soit facile. Parce que la Bibliothèque nationale doit être au service de ses usagers plutôt que ses usagers à la disposition de la Bibliothèque nationale. Parce que le patrimoine conservé au nom de la nation avec l'argent de la nation est la propriété de tous les citoyens. De plus, l'accès aux ressources documentaires doit être égal pour tous. C'est dire qu'il ne doit être prohibitif pour personne.

Il apparaît cependant que l'entrée de la Bibliothèque doit être payante aussi bien pour les non-chercheurs que pour les chercheurs. Parce que tout service au public se paie. Parce que - sans même parler de la reproduction, de l'utilisation de postes de consultation audiovisuels informatisés et de la location de loges de travail - la richesse même des fonds documentaires disponibles et des bases de données bibliographiques consultables est un service supplémentaire que n'offrira aucune autre bibliothèque. Parce qu'il faut tenir compte des redevances que la Bibliothèque elle-méme devra verser sur un certain nombre de documents. Parce qu'il faut éviter l'invasion et le mauvais emploi de la Bibliothèque par un public qui ne serait ni motivé ni qualifié, et en réserver l'usage à ceux qui, ne pouvant trouver ailleurs une documentation et des services équivalents, seront jugés aptes à en tirer le meilleur parti. Parce que, dans le domaine culturel comme ailleurs, tout ce qui est donné est dévalué, tout ce qui est gratuit est méprisé.

\section{Vers l'ouverture...}

Le déménagement des fonds entre Richelieu et Tolbiac devrait se dérouler sur une période de 15 mois environ, à compter de mai 1995 . II respectera le délai d'un mois maximum d'incommunicabilité du document pendant son transfert entre les deux sites. Le déménagement des fonds acquis par l'Établissement public de la Bibliothèque de France (EPBF) et stockés à Ivry se fera sur la même période; ils seront déposés dans les salles de lecture d'ici à octobre 1995.

En toute hypothèse, l'établissement public chargé de l'exploitation devrait prendre réception du bâtiment dès janvier 1995 et assurer, tout au long de l'année 1995, l'achèvement de la préfiguration et la préparation de l'ouverture et de l'exploitation de la nouvelle bibliothèque. Cette préparation comprendra notamment la formation des personnels aux fonctions classiques ou nouvelles de la Bibliothèque, en particulier la maîtrise des applications informatiques, des espaces et des équipements de salles, des magasins et des ateliers. L'arrivée des personnels de la
Bibliothèque nationale et de ceux provenant de recrutements nouveaux sera échelonnée sur les années 1994-1995. L'ouverture de la nouvelle bibliothèque se fera de façon progressive, à compter de janvier 1996.

\section{Un atout majeur}

La nécessité de la novation fut discutée, elle ne l'est plus. Les craintes qui s'exprimaient se sont dissipées comme des fumées. Ceux-mêmes qui redoutaient et condamnaient l'ouverture à tous les publics préconisent kun accès aisé au commun des mortels».

II apparaît tout d'abord qu'à son niveau, avec ses fins propres, la Bibliothèque de France sera un instrument de démocratisation culturelle comme l'ont été en leur temps les Maisons de la Culture ou le Grand Louvre. En offrant une assise nouvelle à la recherche, elle assurera une relance des recrutements et des qualifications, donc de la profession de bibliothécaire qu'elle irriguera et revivifiera. Encyclopédique, multimédiatique, informatisée, couronnant l'édifice des bibliothèques à partir du socle incomparable que constitue l'héritage de la Nationale, elle dotera la France d'un outil scientifique adapté à son rayonnement intellectuel, atout majeur qui lui permettra de conserver ou de retrouver son rang dans la compétition internationale et contribuera à dégager un solde positif dans la balance des comptes intellectuels. A quelle oeuvre plus utile aurait-on pu consacrer l'argent public?

C'est une grande chance de construire une nouvelle bibliothèque nationale. Chance pour l'architecte qui laisse une telle trace de lui-même et du $X X^{e}$ siècle dans le paysage de Paris. Chance pour l'équipe qui a l'honneur et la charge de mener une telle entreprise au service de la collectivité. Chance pour le pays qui bénéficiera des résultats d'un tel effort. 